\title{
Comparing Three Different Modified Sitting Positions for Ease of Spinal Needle Insertion in Patients Undergoing Spinal Anesthesia
}

\author{
Sussan Soltani Mohammadi, ${ }^{1,}$ Mohammadreza Piri, ${ }^{1}$ and Alireza Khajehnasiri ${ }^{1}$ \\ ${ }^{1}$ Department of Anesthesiology, Critical Care and Pain Medicine, Tehran University of Medical Sciences, Dr Shariati Hospital, Tehran, Iran \\ "Corresponding author: Sussan Soltani Mohammadi, MD, Department of Anesthesiology, Critical Care and Pain Medicine, Dr Shariati Hospital, North Kargar St, Ale-Ahmad \\ Highway, Tehran, Iran. Tel: +98-9121226683, Fax: +98-2188633039, E-mail: soltanmo@sina.tums.ac.ir \\ Received 2017 June 21; Revised 2017 August 10; Accepted 2017 October 08.
}

\begin{abstract}
Background: The most important point for performing a neuroaxial block in a sitting position is reducing lumbar lordosis, resulting in easier access to interspinous space and dura mater. There are a few studies comparing 2 different sitting positions including a traditional sitting position (TSP) versus forward bending or hamstring stretch position (HSP) as well as TSP versus squatting position (SP) for reversing the lumbar lordosis and improving access to intervertebral space for neuroaxial block.

Objectives: We compared 3 different sitting positions including traditional sitting position vs. hamstring stretch position vs. squatting position and hypothesized that squatting position reverses the lumbar lordosis and reduces the number of spinal needle bone contacts more than TSP and HSP.

Methods: A total of Thirty hundred and sixty ASA class I or II patients aged 18 to 60 years were scheduled for elective surgeries under spinal anesthesia were randomized into 3 groups. Our primary endpoint was the number of spinal needle-bone contacts and our secondary endpoint was ease of needle insertion or space identification.

Results: Demographic data were statistically different between the study groups. There was no statistical difference between the study groups regarding the number of needle bone contacts and the ease of finding intervertebral space $(\mathrm{P}=0.63, \mathrm{P}=0.56$, respectively).

Conclusions: There was no statistical difference between the TSP, HSP, and SP regarding the number of needle bone contacts and the ease of finding of intervertebral space. In this regard, each of these 3 positions can be used as an alternative sitting position for administration of spinal anesthesia.
\end{abstract}

Keywords: Hamstring Stretch Position, Spinal Anesthesia, Spinal Needle, Squatting Position, Traditional Sitting Position

\section{Background}

Patient positioning during administration of spinal anesthesia is very important. Poor positioning may cause repeated spinal needle insertions and increase the risk of back pain, post-dural puncture headache (PDPH), epidural hematoma, and neural trauma. The sitting position is frequently used for patients undergoing spinal anesthesia, especially when low lumbar and sacral levels of sensory anesthesia are needed for the surgical procedure. The most important point for performing a neuroaxial block in a sitting position is reducing lumbar lordosis, resulting in easier access to the intervertebral space; on the other hand, lumbar flexion pushes the theca sac into a more superficial position (1-13).

There are a few studies regarding different modified sitting positions for this purpose. In a study by Tashayod et al. a kind of modified sitting position with maximum extension of knees, adduction of hips, and forward bending (hamstring stretch position, HSP) was described as more effective in reducing lordosis of lumbar spine and making spinal puncture easier. Even moderate passive knee extension of a patient in a sitting position can increase hamstring tension, tilt the pelvis, and reduce lumbar lordosis (2).

In a study by Fisher et al., 205 patients in TSP were compared with 201 patients in HSP regarding the number of needle-bone contact during epidural labor analgesia. The number of needle-bone contacts were the same in both groups (3).

In another study by Soltani Mohammadi et al., 222 patients, in squatting position (SP), were compared with 230 patients in TSP who were scheduled for elective lower abdomen or lower extremity surgeries. The number of spinal needle-bone contacts was lower in the SP group, nonetheless ease of needle insertion or space identification was the same in both groups (4). Manggala et al., compared the Crossed-Leg Sitting Position (CLSP) with TSP in patients undergoing urologic surgery and concluded that there was no significant difference in success rate of needle placement between the 2 groups and they suggested that the CLSP can be used as an alternative sitting position for ad- 
ministration of spinal anesthesia (5).

We conducted a study to compare 3 different sitting positions including HSP, TSP, and SP to conclude which position can effectively reduce spinal needle-bone contact by reducing lordosis of lumbar spine and improving needle insertion or space identification. Since the study of Manggala and coworkers was published when we had enrolled and collected our data, the CLSP was not included in our study design. Our primary goal was to minimize needle bone contact and the secondary goal was ease of needle insertion/space identification.

\section{Methods}

This study was conducted at the Dr. Shariati hospital, Tehran University of Medical Sciences from January to April 2016. The study protocol conformed to the ethical guidelines of the 1989 Declaration of Helsinki. Ethical approval for this study was provided by the ethics committee of Tehran University of Medical Sciences.

Patients were informed about the proposed position for the procedure during the pre-anesthetic visit. A written informed consent was obtained separately before the operation.

A total of 360 Three hundred and sixty ASA class I or II patients aged 18 to 60 years, scheduled for elective lower abdominal or lower extremity surgeries under spinal anesthesia, were enrolled in the study. Randomization was based on computer-generated codes and was concealed in envelopes, opened by the block performer before the spinal puncture. The cxclusion criteria included any contraindications to neuroaxial block, pregnancy, BMI > 35, lumbar surgical scar, and obvious lumbar scoliosis. All patients had an intravenous (IV) infusion placed and were given isotonic saline $3 \mathrm{~mL} / \mathrm{kg}$ and $50 \mu \mathrm{g}$ Fentanyl before spinal anesthesia. Standard monitoring was used during spinal anesthesia. All spinal blocks were performed by 2 anesthesiologists who had experienced more than 400 spinal procedures in the TSP or SP. The anesthesiologist sat on a stool at a level to keep the interspinous space at the level of his or her eyes. Then, block performers used a 25gauge needle with a length of $3.8 \mathrm{~cm}$ for local anesthetic injection, followed by a 25-gauge Quincke needle by midline approach at L2 - L3 or L3 - L4 interspace. An anesthesiology resident recorded weight, height, and the surface landmarks graded by a block performer as: easy, difficult, or impossible to palpate the lumbar spinous processes, while the patient was positioned according to the allocation group.

For TSP, patients flexed their knees approximately $90^{\circ}$, adducted their hips, and put their feet on a stool; the height of the bed was adjusted to provide optimal hip and lumbar flexion (Figure 1). In SP, patients sat with their lower extremities fully flexed at the hip and knee joints while hugging their knees. In addition, both buttock and plantar surfaces of the feet were supported by the bed and forward bending (Figure 2). In HSP, patients stretched their legs on the operating table with maximum extension of knees, adduction of hips, and forward bending. In each group, "maximum" was the greatest amount that a patient could tolerate (Figure 3 ).

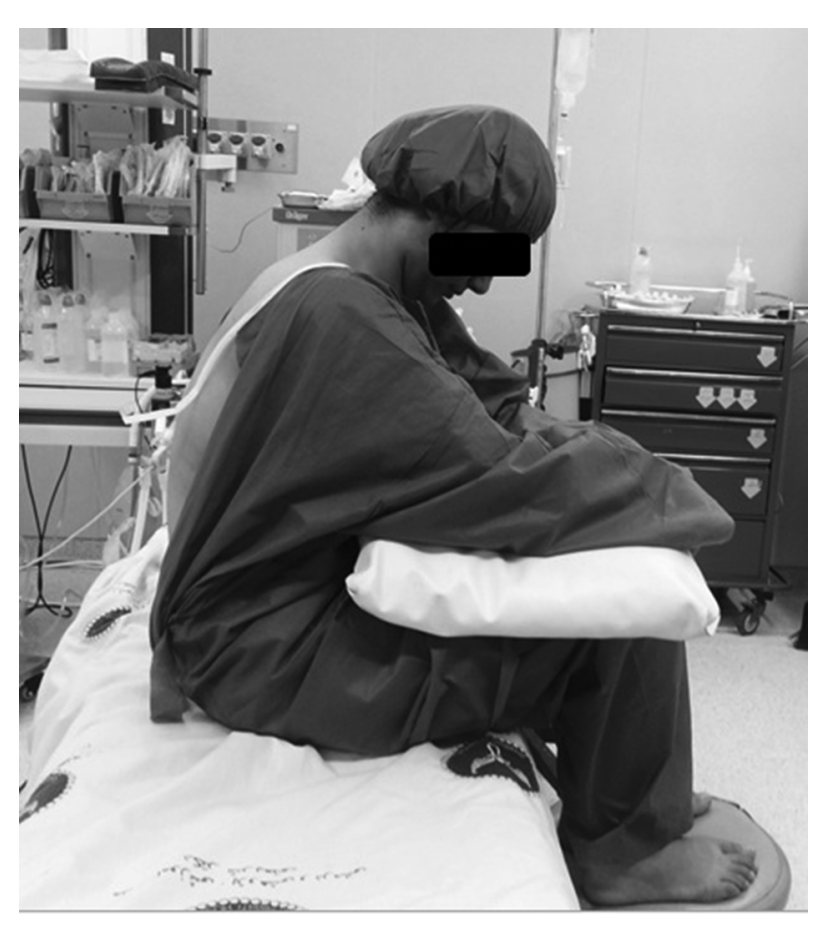

Figure 1. Depicture of Patient in Traditional Sitting Position

The spinal procedures were performed to improve needle insertion or space identification and minimize spinal needle-bone contacts. A spinal needle-bone contact was defined as spinal needle contact against the bone, which prevented further passage. All spinal needle-bone contacts were recorded. The study was complete whenever the subarachnoid space was confirmed by observation of free flow of CSF. When there was no CSF in the needle hub or there was only a small amount of CSF with poor flow, the needle was rotated clockwise $90^{\circ}$ and had to wait for 5 seconds. The sequence of rotation continued for another 3quadrant rotation of $90^{\circ}$ and waited 5 seconds after each rotation. Despite this maneuver, if there was absence of CSF or its free flow, the needle was further advanced approximately by $2 \mathrm{~mm}$. The block performer was not allowed to perform a new puncture site and was restricted to pull back the needle just to the subcutaneous tissue. When 


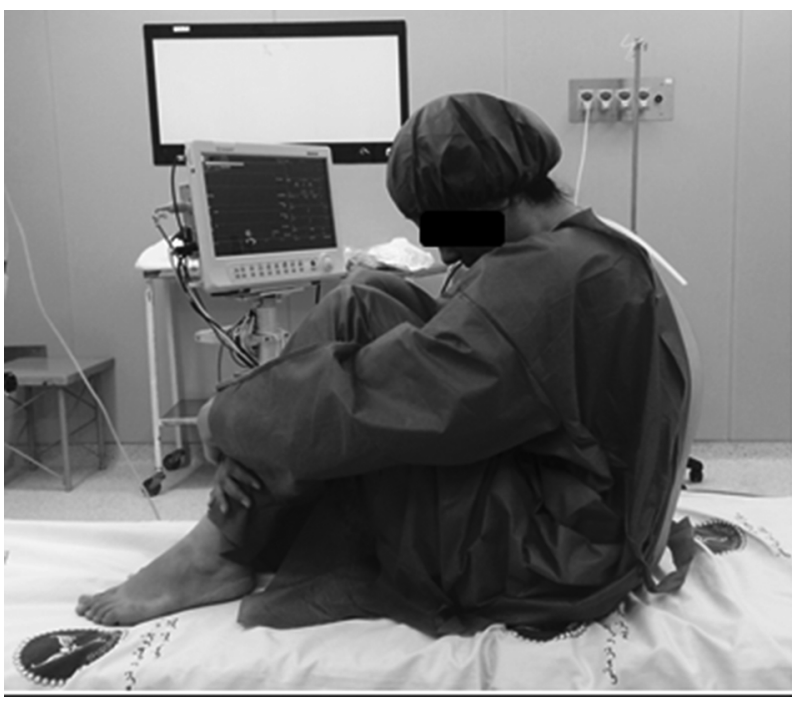

Figure 2. Depicture of Patient in Squatting Position

a bone was encountered during any of above-mentioned attempts, the needle was withdrawn just below the skin level followed by redirection with a more cephalad angulation. If more than 5 spinal needle-bone contacts occurred the case was recorded as a failure of the position and the study was stopped.

\subsection{Statistical Analysis}

According to previous studies, the success rate of needle insertion without bone contact was 50\% in TSP and 70\% in SP. Presuming that HSP would increase this proportion to $85 \%$, one would need to enroll 110 patients in each group for the results to be statistically significant at a power of $80 \%$ with a level of confidence of $95 \%$. Estimating that $10 \%$ of patients may drop out of the study due to different reasons, the sample size was increased to 120 patients in each group. In this regard, with $\alpha=0.05$ and power $80 \%$, we can estimate the difference between SP and TSP with power 90\% and between TSP and HSP with power 95\%.

Data was analyzed by using SPSS version 23 (SPSS Inc., Chicago, IL). Normal distribution of data was checked by the Kolmogorov Smirnov test. Independent sample t-test and Chi-square test were used for comparing demographic data. ANOVA and Post hoc Tukey test were used for comparing a number of needle bone contact and ease of intervertebral space identification. $\mathrm{P} \leq 0.05$ was considered statistically significant.

\section{Results}

A total of 371 patients were chosen through consecutive sampling for spinal anesthesia according to inclusion criteria. There were 11 patients who dropped out, 5 patients of SP group discontinued the position because of discomfort, and 4 patients in the TSP group as well as 2 patients in HSP group were excluded because of failed spinal needle placement. Finally, 360 patients were randomized into 3 equal groups $(\mathrm{n}=120)$.

Demographic data regarding age, sex, weight, and BMI were not statistically different between the study groups (Table 1, P > 0.05).

Table 1. Comparing Demographic Data Between the Study Groups $(\mathrm{N}=120)^{\mathrm{a}}$

\begin{tabular}{lccc}
\hline Variable & Squatting & Traditional Sitting & Hamstring Stretch \\
\hline Age, $\mathbf{y}$ & $40.4 \pm 14.0$ & $41.2 \pm 13.6$ & $40.7 \pm 15.3$ \\
Sex, M/F & $55 / 65$ & $57 / 63$ & $59 / 61$ \\
\hline Weight, $\mathbf{~ g ~}$ & $69.0 \pm 10.4$ & $73.7 \pm 11.1$ & $74.8 \pm 12.1$ \\
\hline Height, $\mathbf{~ C m}$ & $166.3 \pm 9.2$ & $167.1 \pm 10.7$ & $168.8 \pm 7.9$ \\
\hline BMI, Kg/m & $24.8 \pm 2.7$ & $26.2 \pm 3.4$ & $26.1 \pm 3.6$ \\
\hline
\end{tabular}

${ }^{\mathrm{a}}$ Data are presented as mean $\pm \mathrm{SD}, \mathrm{P}>0.05$.

One patient in the SP group, 2 patients in the TSP group, and 1 in HSP group were considered as "failures" ( $>5$ needle-bone contacts), however, there was no statistical difference between the study groups regarding the number of needle bone contacts $(P=0.63$, Figure 4$)$.

There was no statistical difference between the study groups regarding the ease of finding of intervertebral space. However, it was easier in the SP and TSP, group respectively (Table 2, $\mathrm{P}>0.05$ ).

\section{Discussion}

The sitting position is one of the popular positions for spinal anesthesia, especially when low lumbar and sacral levels of sensory anesthesia are needed. There are different types of modified sitting positions for this procedure. In this study, 3 different sitting positions were compared and there was no statistical difference between the traditional sitting position, hamstring stretch position, and squatting position regarding the number of needle-bone contacts and the ease of finding intervertebral space. In this regard, each of these 3 positions can be used as an alternative sitting position for administration of spinal anesthesia.

In a study by Fisher et al., on 406 parturient patients, scheduled for epidural analgesia for labor pain, the number of needle bone contact was equal in both TSP and HSP 


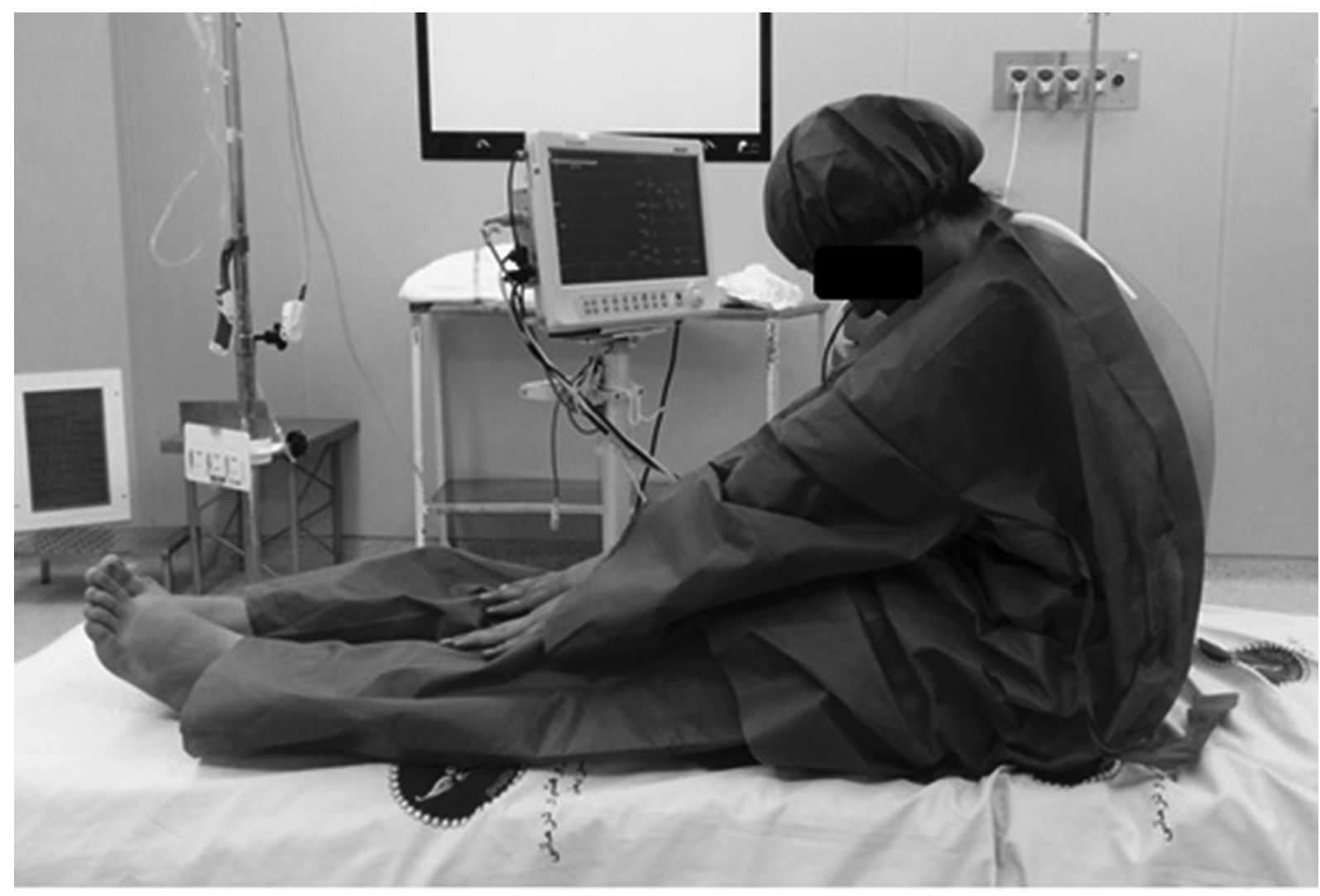

Figure 3. Depicture of Patient in Hamstring Stretch Position

Table 2. Comparing the Study Groups Regarding the Ease of Identifying Intervertebral Space

\begin{tabular}{|c|c|c|c|c|}
\hline Variable & Easy & Difficult & Impossible & Total Number \\
\hline Squatting position & 101 & 19 & 0 & 120 \\
\hline Traditional sitting position & 97 & 23 & 0 & 120 \\
\hline Hamstring stretch position & 64 & 52 & 4 & 120 \\
\hline
\end{tabular}

${ }^{\mathrm{a}} \mathrm{P}$ value $>0.05$ between the groups, Date are presented as number of patients.

groups, which was similar to our study, however, they did not include the squatting position in their allocation because of pregnancy, which could had affected the results (3).

In a study by Soltani Mohammadi et al., 260 patients aged 18 to 75 years, who were scheduled for elective surgeries under spinal anesthesia, in 2 different traditional sitting and squatting positions were compared. They found that the number of spinal needle-bone contacts was lower in the SP group compared to the TSP group. Nonetheless, the ease of needle insertion or space identification was the same in the both groups. Their findings regarding space identification was similar to our study; however, regard- ing needle-bone contact, we had no difference between the study groups, which may be due to narrower range of age in our study (4).

Our study had several limitations including: lack of blinding due to apparent differences between the interventions, exclusion of morbidly obese patients, pregnant patients, and patients older than 60 years, most of whom cannot tolerate squatting or hamstring stretch position. The last limitation was the parameters that were measured subjectively. As a whole, there was no statistical difference between the TSP, HSP, and SP regarding the number of needle bone contacts and the ease of spinal needle insertion/finding of intervertebral space. 


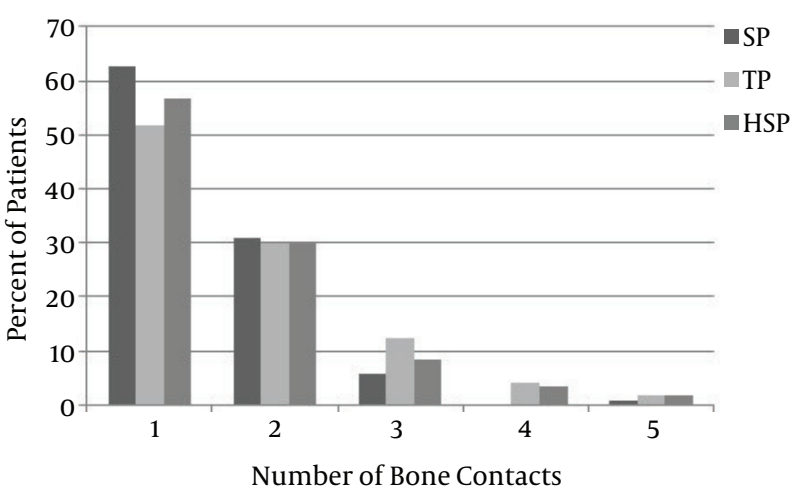

Figure 4. Comparing Study Groups Regarding the Number of Needle Bone Contacts

We suggest further studies with larger samples such as including the crossed leg sitting position as well as patients with larger BMI and geriatric patients to compare the success rate of spinal needle placement in difficult cases.

\section{Acknowledgments}

We appreciate the cooperation of all physicians, nurses, and anesthesia staff that helped us to conduct the study.

\section{Footnotes}

Authors' Contribution: Authors' contribution is as follows, study concept and design, Dr. Sussan Soltani Mohammadi; data collection, Dr. Mohammadreza Piri; data analysis and manuscript preparation, Dr. Sussan Soltani Mohammadi; critical revision of the manuscript for important intellectual content, Dr. Sussan Soltani Mohammadi, Dr Alireza Khajehnasiri.

Financial Disclosure: We had no financial interests related to the materials in the manuscript.

\section{References}

1. Biswas BK, Agarwal B, Bhattarai B, Dey S, Bhattacharyya P. Straight versus flex back: Does it matter in spinal anaesthesia? Indian J Anaesth. 2012;56(3):259-64. doi:10.4103/0019-5049.98772. [PubMed: 22923825].

2. Tashayod ME, Tamadon S. Spinal block in sitting position without moving the legs. Middle East JAnaesthesiol. 1980;5(8):529-33. [PubMed: 7253944].

3. Fisher KS, Arnholt AT, Douglas ME, Vandiver SL, Nguyen DH. A randomized trial of the traditional sitting position versus the hamstring stretch position for labor epidural needle placement. Anesth Analg. 2009;109(2):532-4. doi: 10.1213/ane.0b013e3181ac6c79. [PubMed: 19608828].

4. Soltani Mohammadi S, Hassani M, Marashi SM. Comparing the squatting position and traditional sitting position for ease of spinal needle placement: a randomized clinical trial. Anesth Pain Med. 2014;4(2):e13969. doi: 10.5812/aapm.13969. [PubMed: 24790901].

5. Manggala SK, Tantri AR, Satoto D. Comparison of Successful Spinal Needle Placement Between Crossed-Leg Sitting Position and Traditional Sitting Position in Patients Undergoing Urology Surgery. Anesth Pain Med. 2016;6(4):e39314. doi: 10.5812/aapm.39314. [PubMed: 27843785].

6. Garcia AM, Martí A, Rodriguez PM, Villamor M, Martínez A, Moral MV. Predictors of difficulty in neuroaxial block: A prospective study. Eur J Anaesthesiol. 2011;28:110. doi:10.1097/00003643-201106001-00351.

7. de Filho GR, Gomes HP, da Fonseca MH, Hoffman JC, Pederneiras SG, Garcia JH. Predictors of successful neuraxial block: a prospective study. Eur J Anaesthesiol. 2002;19(6):447-51. doi: 10.1097/00003643200206000-00008. [PubMed:12094920].

8. Tanaka K, Irikoma S, Kokubo S. Identification of the lumbar interspinous spaces by palpation and verified by X-rays. Braz J Anesthesiol. 2013;63(3):245-8. doi: 10.1016/S0034-7094(13)70224-1. [PubMed: 23683445].

9. Fettes PD, Jansson JR, Wildsmith JA. Failed spinal anaesthesia: mechanisms, management, and prevention. BrJAnaesth. 2009;102(6):73948. doi: 10.1093/bja/aep096. [PubMed: 19420004].

10. Sprung J, Bourke DL, Grass J, Hammel J, Mascha E, Thomas P, et al. Predicting the difficult neuraxial block: a prospective study. Anesth Analg. 1999;89(2):384-9. [PubMed:10439752].

11. Atallah MM, Demian AD, Shorrab AA. Development of a difficulty score for spinal anaesthesia. Br J Anaesth. 2004;92(3):354-60. doi: 10.1093/bja/aeh073. [PubMed:14742333].

12. Kim JH, Song SY, Kim BJ. Predicting the difficulty in performing a neuraxial blockade. Korean J Anesthesiol. 2011;61(5):377-81. doi: 10.4097/kjae.2011.61.5.377. [PubMed: 22148085].

13. Movasseghi G, Hassani V, Mohaghegh MR, Safaeian R, Safari S, Zamani $\mathrm{MM}$, et al. Comparison between spinal and general anesthesia in percutaneous nephrolithotomy. Anesth Pain Med. 2014;4(1):e13871. doi: 10.5812/aapm.13871. [PubMed: 24660147]. 